\title{
A Simple Benchmark Problem for the Numerical Methods of the Cahn-Hilliard Equation
}

\author{
Yibao Li, ${ }^{1}$ Chaeyoung Lee, ${ }^{2}$ Jian Wang, ${ }^{3}$ Sungha Yoon, ${ }^{2}$ Jintae Park, ${ }^{2}$ and Junseok Kim (iD ${ }^{2}$ \\ ${ }^{1}$ School of Mathematics and Statistics, Xi'an Jiaotong University, Xi'an 710049, China \\ ${ }^{2}$ Department of Mathematics, Korea University, Seoul 02841, Republic of Korea \\ ${ }^{3}$ School of Mathematics and Statistics, Nanjing University of Information Science and Technology, Nanjing, 210044, China
}

Correspondence should be addressed to Junseok Kim; cfdkim@korea.ac.kr

Received 12 August 2020; Revised 19 February 2021; Accepted 27 February 2021; Published 9 March 2021

Academic Editor: Manuel De la Sen

Copyright (C) 2021 Yibao Li et al. This is an open access article distributed under the Creative Commons Attribution License, which permits unrestricted use, distribution, and reproduction in any medium, provided the original work is properly cited.

\begin{abstract}
We present a very simple benchmark problem for the numerical methods of the Cahn-Hilliard $(\mathrm{CH})$ equation. For the benchmark problem, we consider a cosine function as the initial condition. The periodic sinusoidal profile satisfies both the homogeneous and periodic boundary conditions. The strength of the proposed problem is that it is simpler than the previous works. For the benchmark numerical solution of the $\mathrm{CH}$ equation, we use a fourth-order Runge-Kutta method (RK4) for the temporal integration and a centered finite difference scheme for the spatial differential operator. Using the proposed benchmark problem solution, we perform the convergence tests for an unconditionally gradient stable scheme via linear convex splitting proposed by Eyre and the Crank-Nicolson scheme. We obtain the expected convergence rates in time for the numerical schemes for the one-, two-, and three-dimensional $\mathrm{CH}$ equations.
\end{abstract}

\section{Introduction}

We present a very simple benchmark problem for the numerical methods of the following Cahn-Hilliard $(\mathrm{CH})$ equation [1-3]:

$$
\frac{\partial \phi(x, t)}{\partial t}=\Delta\left[F^{\prime}(\phi(x, t))-\varepsilon^{2} \Delta \phi(x, t)\right], \quad(x, t) \in \Omega \times(0, T],
$$

where $\Omega \subset \mathbb{R}^{d}(d=1,2,3)$ is a bounded domain, $\phi(x, t)$ is a compositional field, $F(\phi)=0.25\left(\phi^{2}-1\right)^{2}$, and $\varepsilon$ is a positive constant. The $\mathrm{CH}$ equation was proposed for a model of the spinodal decomposition in a binary mixture and has been used to model many scientific phenomena such as topology optimization [4], phase separation [5-7], image processing [8], two-phase fluid flows [9, 10], crystal model [11], tumor growth $[12,13]$, and microstructure formations (see [14] for the basic principles and practical applications and [3] for the physical, mathematical, and numerical derivations of the $\mathrm{CH}$ equation).
There have been many research papers regarding the accurate numerical methods for the $\mathrm{CH}$ equation [15-18] (see the references therein for more details). In particular, a comparison study among various numerical methods for the $\mathrm{CH}$ equation was conducted on the perspective of stability and efficiency [19]. A test set developed to verify models, algorithms, or the accuracy of numerical methods is called "benchmark problem" [20]. However, there are only few benchmark problems for validating the accuracy of the proposed numerical methods. Recently, the authors in [21] proposed a benchmark problem for the two- and threedimensional $\mathrm{CH}$ equations. The benchmark problems were the shrinking annulus and spherical shell in the two- and three-dimensional cases, respectively. They used the explicit Euler scheme with a very fine time step size, $\Delta t<10^{-5}$. The authors in [22] presented four benchmark problems for the Allen-Cahn (AC) and $\mathrm{CH}$ equations. The benchmark is the time $T$ at which the value at a point in the domain changes from negative to positive. The authors in [20] presented two benchmark problems for phase-field models of solute diffusion and phase separation. Recently, a verification method 
for the convergence rates of the numerical solutions for wellknown parabolic partial differential equations was proposed in [23].

Typically, benchmark testing is essential for comparing the performance of a method. However, many researchers conduct self-tests using their own methods in most cases. There is a problem such that an error may occur relatively compared to the actual solution because an approximated solution converges to a numerical solution via an employed numerical method although convergence of a solution through numerical analysis is guaranteed. Moreover, most of these testing schemes are often implicit methods, which have relatively low accuracy compared to the results of long time simulation of explicit methods with a small time step. Therefore, the main purpose of this paper is to present a very simple benchmark problem for the numerical methods of the $\mathrm{CH}$ equation, which does not employ the self-test but a classical explicit method for the temporal discretization. The strength of the proposed problem is that it is simpler than the previous works [20-22]. Furthermore, the benchmark problem can be applied to both Neumann and periodic boundary conditions.

The contents of this paper are summarized as follows. In Section 2, a numerical solution is presented. In Section 3, numerical results are shown. Conclusions are made in Section 4.

\section{Numerical Solution}

We consider two discretization schemes to the $\mathrm{CH}$ equation for benchmark problem; one is the linearly stabilized splitting scheme (LSS) proposed by Eyre [24] which is firstorder accurate in time and the other is the Crank-Nicolson (CN) scheme [25] which is second-order in time. In order to perform a benchmark test for these two methods, we first need to obtain a benchmark numerical solution. To obtain the benchmark solution, we consider the one-dimensional $\mathrm{CH}$ equation:

$$
\begin{aligned}
\phi_{t}(x, t)= & {\left[F^{\prime}(\phi(x, t))-\varepsilon^{2} \phi_{x x}(x, t)\right]_{x x}, } \\
& (x, t) \in(0,1) \times(0, T],
\end{aligned}
$$

with the homogeneous Neumann boundary condition $\phi_{x}(0, t)=\phi_{x}(1, t)=\phi_{x x x}(0, t)=\phi_{x x x}(1, t)=0$. Let $h=1 /$ $N_{x}$ be the uniform spatial step size, $x_{i}=(i-0.5) h$ for $1 \leq i \leq N_{x}$, and $t_{n}=n \Delta t_{\mathrm{ref}}$, where $\Delta t_{\mathrm{ref}}=T / N_{t}$ is the reference time step and $T$ is the final time. We define $\phi_{i}^{n}$ as an approximation of $\phi\left(x_{i}, t_{n}\right)$. Now, we discretize equation (2) in time using a fourth-order Runge-Kutta method (RK4) [26] as follows:

$$
\begin{aligned}
\alpha_{i}^{n} & =\Delta t_{\mathrm{ref}} \Delta_{h}\left[F^{\prime}\left(\phi_{i}^{n}\right)-\varepsilon^{2} \Delta_{h} \phi_{i}^{n}\right], \\
\beta_{i}^{n} & =\Delta t_{\mathrm{ref}} \Delta_{h}\left[F^{\prime}\left(\phi_{i}^{n}+0.5 \alpha_{i}^{n}\right)-\varepsilon^{2} \Delta_{h}\left(\phi_{i}^{n}+0.5 \alpha_{i}^{n}\right)\right], \\
\gamma_{i}^{n} & =\Delta t_{\mathrm{ref}} \Delta_{h}\left[F^{\prime}\left(\phi_{i}^{n}+0.5 \beta_{i}^{n}\right)-\varepsilon^{2} \Delta_{h}\left(\phi_{i}^{n}+0.5 \beta_{i}^{n}\right)\right], \\
\delta_{i}^{n} & =\Delta t_{\mathrm{ref}} \Delta_{h}\left[F^{\prime}\left(\phi_{i}^{n}+\gamma_{i}^{n}\right)-\varepsilon^{2} \Delta_{h}\left(\phi_{i}^{n}+\gamma_{i}^{n}\right)\right], \\
\phi_{i}^{n+1} & =\phi_{i}^{n}+\frac{1}{6}\left(\alpha_{i}^{n}+2 \beta_{i}^{n}+2 \gamma_{i}^{n}+\delta_{i}^{n}\right), \quad \text { for } i=1,2, \ldots, N_{x},
\end{aligned}
$$

where $\Delta_{h} \psi_{i}=\left(\psi_{i-1}-2 \psi_{i}+\psi_{i+1}\right) / h^{2}$ is the standard discrete Laplacian with $\psi_{0}=\psi_{1}$ and $\psi_{N_{x}+1}=\psi_{N_{x}}$.

\section{Numerical Results}

3.1. Convergence and Stability Tests. First, we conduct a traditional convergence test. As a concrete example, an initial condition is given by

$$
\phi(x, 0)=0.1 \cos (2 \pi x) \text { on } x \in \Omega=(0,1) .
$$

Note that equation (4) can be used in testing numerical schemes with both the Neumann and periodic boundary conditions. We employ $\varepsilon=16 h /\left(2 \sqrt{2} \tanh ^{-1}(0.9)\right) \approx 0.03$, which is the approximate transition layer width [27]. Table 1 shows the rate of convergence. We measure the accuracy of time at the final time $T$ using $\left\|e^{\Delta t, 0.5 \Delta t}\right\|_{2}$ which is defined as follows:

$$
\left\|e^{\Delta t, \Delta t / 2}\right\|_{2}=\sqrt{\frac{1}{N_{x}} \sum_{i=1}^{N_{x}}\left(e_{i}^{\Delta t, \Delta t / 2}\right)^{2}},
$$

where $e_{i}^{\Delta t, \Delta t / 2}$ is an error between the computed solutions using large and small time steps, respectively. More precisely, it is

$$
e_{i}^{\Delta t, \Delta t / 2}=\left|\phi_{i}^{\Delta t}-\phi_{i}^{\Delta t / 2}\right|
$$

In this test, we adopt the space step size as $h=1 / 10$ and the final time as $T=10 h^{4}$. We can confirm that RK4 is of fourth-order in time indeed according to Table 1.

For the next test, we investigate the maximum time step sizes $\Delta t_{c}$ with respect to the spatial step size $h$. Here, we fix $N_{t}=10^{5}$ and other parameters and the initial condition are the same as those described above. Table 2 lists the largest time step sizes satisfying that the numerical solution does not blow up.

Figure 1 depicts the practically stable region and maximum time step size $\Delta t_{c}$ as described in Table 2 . The explicit scheme is theoretically $\Delta t \approx O\left(h^{4}\right)$. 
TABLE 1: Rate of convergence of RK4.

\begin{tabular}{lccccc}
\hline$(\Delta t, \Delta t / 2)$ & $\left(h^{4}, h^{4} / 2\right)$ & Rate & $\left(h^{4} / 2, t h^{4} / 4\right)$ & Rate & $\left(h^{4} / 4, h^{4} / 8\right)$ \\
\hline$\left\|e^{\Delta t, \Delta t / 2}\right\|_{2}$ & $4.378 \mathrm{e}-11$ & 4.030 & $2.681 \mathrm{e}-12$ & 4.097 & $1.567 \mathrm{e}-13$
\end{tabular}

TABle 2: Practical maximum time step size $\Delta t_{c}$ with respect to $h$.

\begin{tabular}{lcccc}
\hline$h$ & $1 / 16$ & $1 / 32$ & $1 / 64$ & $1 / 128$ \\
\hline$\Delta t_{c}$ & $4.686 e-5$ & $1.152 e-5$ & $2.810 e-6$ & $7.085 e-7$ \\
\hline
\end{tabular}

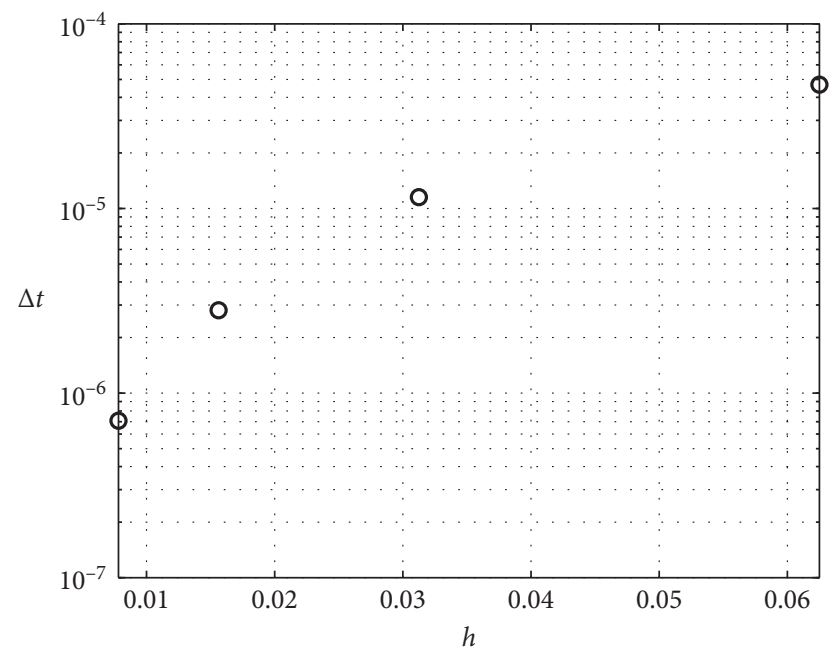

Figure 1: Practical maximum time step size $\Delta t_{c}$ with respect to $h$.

Figure 2 represents the CPU times (seconds) with respect to the number of spatial step $N_{x}$, performed on Intel Core i5-6400 CPU at $2.70 \mathrm{GHz}$ with $4 \mathrm{~GB}$ RAM. When we vary $N_{x}$ as $16,32,64$, and 128 with fixed parameters, $\Delta t=(1 / 128)^{4}$ and $N_{t}=10^{7}$, the CPU times are 88.812, 175.468, 354.14, and 708.718. That is, when $N_{x}$ is doubled, the CPU time is approximately doubled.

3.2. Benchmark Problem. Let us consider the benchmark problem for the $\mathrm{CH}$ equation. We adopt equation (4) as an initial condition. For the reference numerical solution of the benchmark problem, we use $h=1 / 128, \Delta t_{\text {ref }}=h^{4}$, and $N_{t}=10^{7}$. Therefore, the final time is $T=N_{t} \Delta t_{\text {ref }}=10^{7} h^{4}$. For the model parameter, we use $\varepsilon=24 h /\left(2 \sqrt{2} \tanh ^{-1}(0.9)\right) \approx 0.045$. Figure 3 shows the initial condition and the reference numerical solution at $t=10^{7} h^{4}$.

3.2.1. One-Dimensional Space. Let us define an error $e(x, T)$ as the difference between $\phi_{\Delta t}$ and $\phi_{\Delta t_{\text {ref }}}$ at the final time $T$, i.e., $e(x, T)=\left|\phi_{\Delta t}(x, T)-\phi_{\Delta t_{\text {ref }}}(x, T)\right|$, where $\phi_{\Delta t}$ and $\phi_{\Delta t_{\text {ref }}}$ are the numerical solution with a time step $\Delta t$ and the reference numerical solution with a time step $\Delta t_{\text {ref }}$, respectively. The one-dimensional LSS and CN schemes to be benchmarked are as follows:

$$
\begin{aligned}
\frac{\phi_{i}^{n+1}-\phi_{i}^{n}}{\Delta t}= & \Delta_{h}\left[\left(\phi_{i}^{n}\right)^{3}-3 \phi_{i}^{n}+2 \phi_{i}^{n+1}-\varepsilon^{2} \Delta_{h} \phi_{i}^{n+1}\right], \\
\frac{\phi_{i}^{n+1}-\phi_{i}^{n}}{\Delta t}= & \frac{1}{2} \Delta_{h}\left[\left(\phi_{i}^{n}\right)^{3}-\phi_{i}^{n}-\varepsilon^{2} \Delta_{h} \phi_{i}^{n}+\left(\phi_{i}^{n+1}\right)^{3}\right. \\
& \left.-\phi_{i}^{n+1}-\varepsilon^{2} \Delta_{h} \phi_{i}^{n+1}\right],
\end{aligned}
$$

where $\Delta t$ is a time step. We solve equations (7) and (8) using a multigrid method $[25,28]$. Note that we use the Gauss-Seidel iterative method in multigrid process combined with Newton's approximation to compute the nonlinear term in equation (8). Figures 4(a) and 4(b) show the errors for the LSS and CN scheme, respectively.

Now, we define the discrete $l_{2}$-norm and the discrete maximum norm as

$$
\begin{aligned}
& \|e\|_{2}=\sqrt{\frac{1}{N_{x}} \sum_{i=1}^{N_{x}} e_{i}^{2},} \\
& \|e\|_{\infty}=\max _{1 \leq i \leq N_{x}} e_{i},
\end{aligned}
$$

respectively. Tables 3 and 4 list the numerical errors and convergence rates of the LSS and those of the CN scheme at $t=10^{7} h^{4}$, respectively. We confirm that both the schemes achieve the expected convergence rates (the first-order for the LSS and the second-order for the CN scheme).

3.2.2. Two-Dimensional Space. Next, we consider the twodimensional version of equations (7) and (8) to the $\mathrm{CH}$ equation on $\Omega=(0,1) \times(0,1)$. Straightforward extensions of the LSS and CN schemes are as follows:

$$
\begin{aligned}
\frac{\phi_{i j}^{n+1}-\phi_{i j}^{n}}{\Delta t}= & \Delta_{h}\left[\left(\phi_{i j}^{n}\right)^{3}-3 \phi_{i j}^{n}+2 \phi_{i j}^{n+1}-\varepsilon^{2} \Delta_{h} \phi_{i j}^{n+1}\right], \\
\frac{\phi_{i j}^{n+1}-\phi_{i j}^{n}}{\Delta t}= & \frac{1}{2} \Delta_{h}\left[\left(\phi_{i j}^{n}\right)^{3}-\phi_{i j}^{n}-\varepsilon^{2} \Delta_{h} \phi_{i j}^{n}\right. \\
& \left.+\left(\phi_{i j}^{n+1}\right)^{3}-\phi_{i j}^{n+1}-\varepsilon^{2} \Delta_{h} \phi_{i j}^{n+1}\right]
\end{aligned}
$$

where $\Delta_{h} \psi_{i j}=\left(\psi_{i-1, j}+\psi_{i+1, j}+\psi_{i, j-1}+\psi_{i, j+1}-4 \psi_{i j}\right) / h^{2}$ and $h=1 / N_{x}=1 / N_{y}=1 / 128$. We denote $\phi\left(x_{i}, y_{j}, t_{n}\right)=\phi((i-$ $0.5) h,(j-0.5) h, n \Delta t)$ by $\phi_{i j}^{n} \quad$ for $i=1, \ldots, N_{x}$ and $j=1, \ldots, N_{y}$. An initial condition is given by a simple extension of equation (4):

$$
\phi(x, y, 0)=0.1 \cos (2 \pi x) \text { on } \Omega .
$$

We can minimize the influence of domain in this way. Therefore, the two-dimensional reference numerical solution is extended from that of the one-dimensional as follows:

$$
\phi_{i j}=\phi_{i}, \quad \text { for } 1 \leq i \leq N_{x}, 1 \leq j \leq N_{y} .
$$

Figures 5(a) and 5(b) show the initial condition and the reference solution at $t=10^{7} h^{4}$, respectively.

We define the discrete $l_{2}$-norm and the discrete maximum norm as 


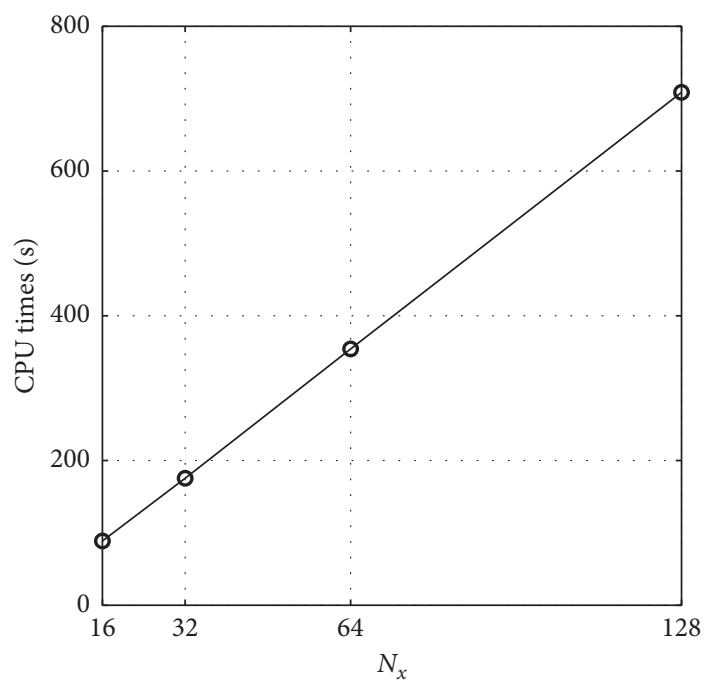

Figure 2: CPU times versus spatial step.

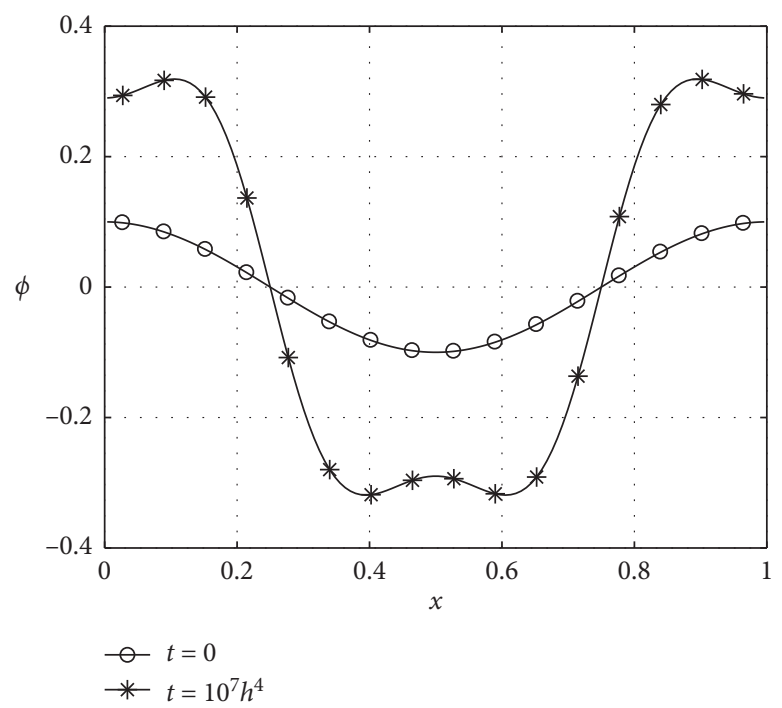

Figure 3: Initial condition and the reference numerical solution at $t=10^{7} h^{4}$.

$$
\begin{aligned}
\|e\|_{2} & =\sqrt{\frac{1}{N_{x} N_{y}} \sum_{i=1}^{N_{x}} \sum_{j=1}^{N_{y}} e_{i j}^{2}}, \\
\|e\|_{\infty} & =\max _{\substack{1 \leq i \leq N_{x} \\
1 \leq j \leq N_{y}}} e_{i j},
\end{aligned}
$$

respectively. Tables 5 and 6 list the errors and convergence rates of the numerical results at $t=10^{7} h^{4}$. From the results, we observe that both the schemes achieve the expected convergence rates (the first-order for LSS (10) and the second-order for $\mathrm{CN}$ scheme (11)).

3.2.3. Three-Dimensional Space. Finally, the two numerical schemes are extended to the three-dimensional $\mathrm{CH}$ equation as follows:

$$
\begin{aligned}
& \frac{\phi_{i j k}^{n+1}-\phi_{i j k}^{n}}{\Delta t}=\Delta_{h}\left[\left(\phi_{i j k}^{n}\right)^{3}-3 \phi_{i j k}^{n}+2 \phi_{i j k}^{n+1}-\varepsilon^{2} \Delta_{h} \phi_{i j k}^{n+1}\right], \\
& \frac{\phi_{i j k}^{n+1}-\phi_{i j k}^{n}}{\Delta t}=\frac{1}{2} \Delta_{h}\left[\left[\left(\phi_{i j k}^{n}\right)^{3}-\phi_{i j k}^{n}-\varepsilon^{2} \Delta_{h} \phi_{i j k}^{n}+\left(\phi_{i j k}^{n+1}\right)^{3}-\phi_{i j k}^{n+1}-\varepsilon^{2} \Delta_{h} \phi_{i j k}^{n+1}\right],\right.
\end{aligned}
$$




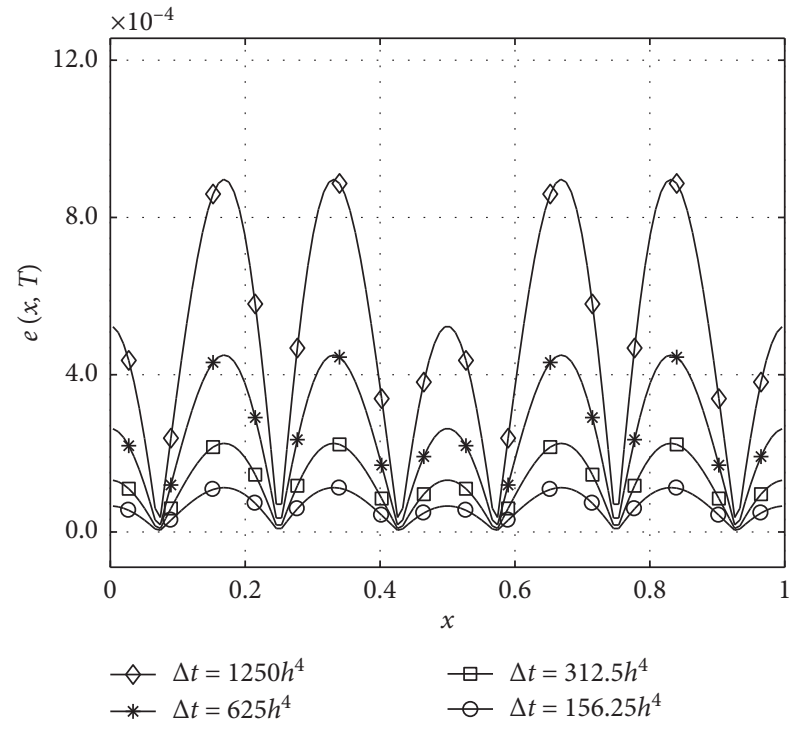

(a)

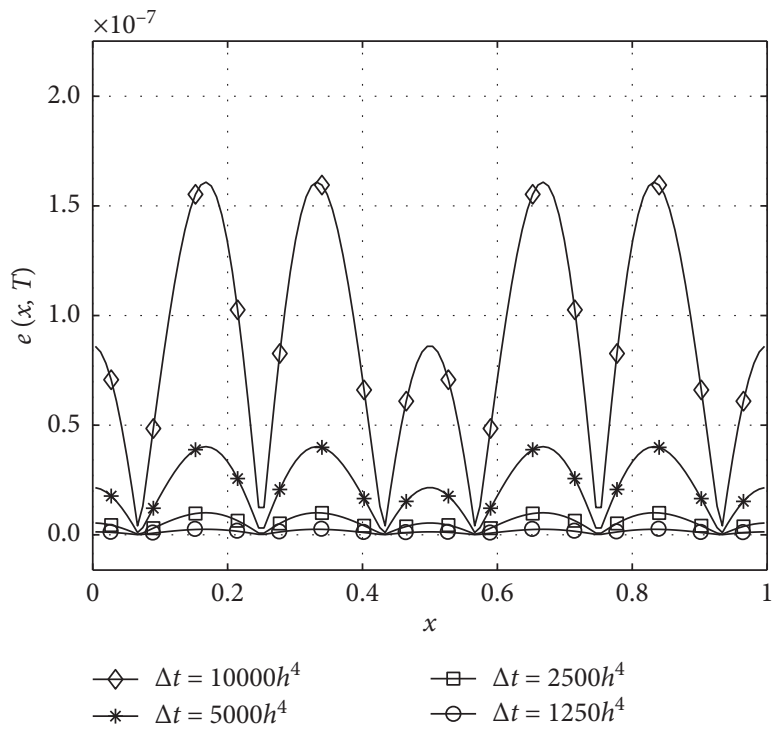

(b)

FIGURE 4: Errors between the reference solution and the solutions evaluated using (a) the LSS and (b) the CN scheme at $t=10^{7} h^{4}$.

TABLE 3: Errors and convergence rates for the numerical results of the LSS at the final time $T=10^{7} h^{4}$. Here, $h=1 / 128$ is used.

\begin{tabular}{lccccccc}
\hline$\Delta t$ & $1250 h^{4}$ & Rate & $625 h^{4}$ & Rate & $312.5 h^{4}$ & Rate & $156.25 h^{4}$ \\
\hline$\|e\|_{2}$ & $5.687 e-4$ & 0.995 & $2.854 e-4$ & 0.997 & $1.430 e-4$ & 0.999 & $7.150 e-5$ \\
$\|e\|_{\infty}$ & $8.970 e-4$ & 0.995 & $4.500 e-4$ & 0.998 & $2.254 e-4$ & 0.999 & $1.128 e-4$ \\
\hline
\end{tabular}

TABLE 4: Errors and convergence rates for the numerical results of the CN scheme at the final time $T=10^{7} h^{4}$. Here, $h=1 / 128$ is used.

\begin{tabular}{lccccccc}
\hline$\Delta t$ & $10000 h^{4}$ & Rate & $5000 h^{4}$ & Rate & $2500 h^{4}$ & Rate & $1250 h^{4}$ \\
\hline$\|e\|_{2}$ & $1.014 e-7$ & 2.000 & $2.540 e-8$ & 2.000 & $6.300 e-9$ & 2.001 & $1.600 e-9$ \\
$\|e\|_{\infty}$ & $1.608 e-7$ & 2.000 & $4.020 e-8$ & 2.000 & $1.010 e-8$ & 2.000 & $2.500 e-9$ \\
\hline
\end{tabular}

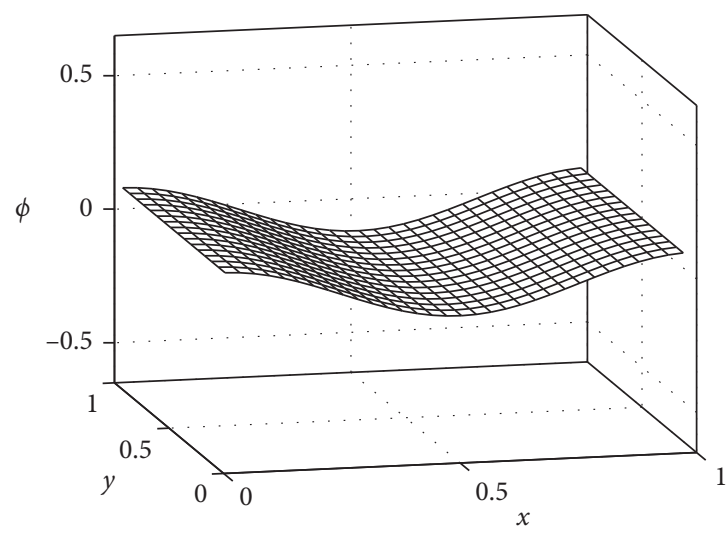

(a)

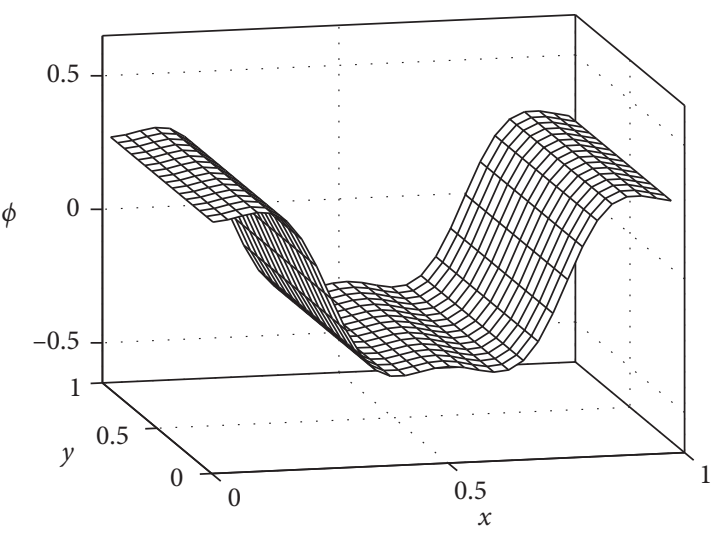

(b)

FIGURE 5: (a, b) The initial condition and the reference solution at $t=10^{7} h^{4}$, respectively.

where $\Delta_{h} \phi_{i j k}=\left(\phi_{i-1, j k}+\phi_{i+1, j k}+\phi_{i, j-1, k}+\phi_{i, j+1, k}+\phi_{i j, k-1}+\right.$ $\left.\phi_{i j, k+1}-6 \phi_{i j k}\right) / h^{2}$. Here, $h=1 / N_{x}=1 / N_{y}=1 / N_{z}=1 / 128$. An initial condition is given by

$$
\phi(x, y, z, 0)=0.1 \cos (2 \pi x) \text { on } \Omega=(0,1)^{3} .
$$

We extend the one-dimensional reference solution to the three-dimensional reference numerical solution:

$$
\phi_{i j k}=\phi_{i}, \quad \text { for } 1 \leq i \leq N_{x}, 1 \leq j \leq N_{y}, 1 \leq k \leq N_{z} \text {. }
$$


TABLE 5: Errors and convergence rates for the numerical results of the LSS at the final time $T=10^{7} h^{4}$. Here, $h=1 / 128$ is used.

\begin{tabular}{lccccccr}
\hline$\Delta t$ & $2500 h^{4}$ & Rate & $1250 h^{4}$ & Rate & $625 h^{4}$ & Rate & $312.5 h^{4}$ \\
\hline$\|e\|_{2}$ & 0.0011 & 0.990 & 0.0006 & 0.995 & 0.0003 & 0.997 & 0.0001 \\
$\|e\|_{\infty}$ & 0.0018 & 0.990 & 0.0009 & 0.995 & 0.0005 & 0.998 & 0.0002 \\
\hline
\end{tabular}

TABLE 6: Errors and convergence rates for the numerical results of the CN scheme at the final time $T=10^{7} h^{4}$. Here, $h=1 / 128$ is used.

\begin{tabular}{lccccccc}
\hline$\Delta t$ & $10000 h^{4}$ & Rate & $5000 h^{4}$ & Rate & $2500 h^{4}$ & Rate & $1250 h^{4}$ \\
\hline$\|e\|_{2}$ & $1.014 e-7$ & 2.000 & $2.540 e-8$ & 2.000 & $6.300 e-9$ & 1.999 & $1.600 e-9$ \\
$\|e\|_{\infty}$ & $1.608 e-7$ & 2.000 & $4.020 e-8$ & 1.999 & $1.010 e-8$ & 1.997 & $2.500 e-9$ \\
\hline
\end{tabular}

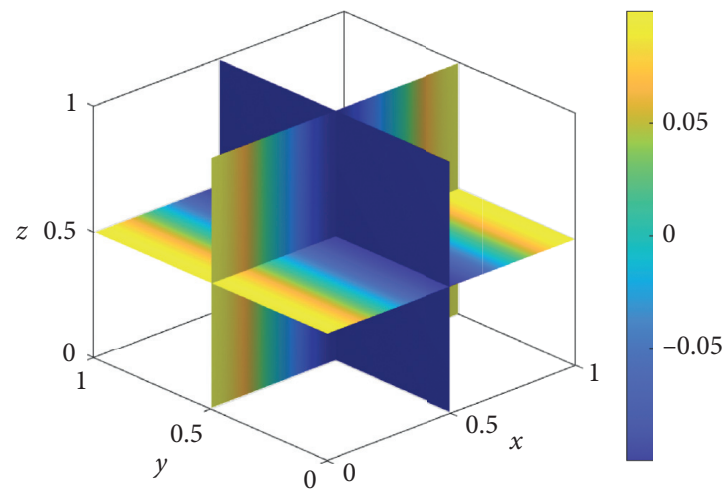

(a)

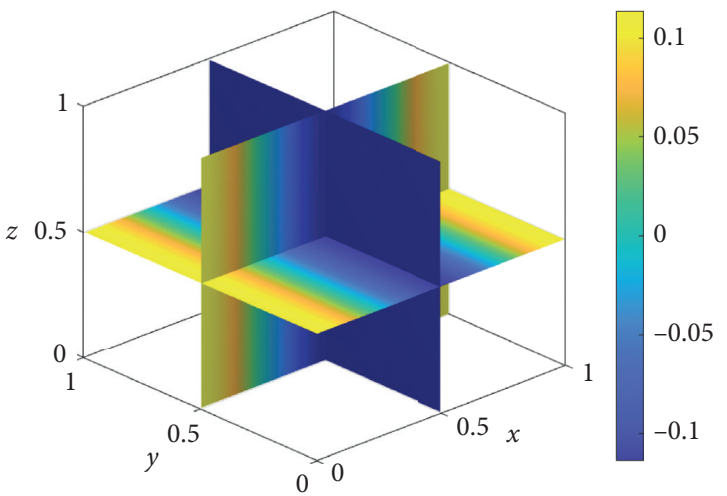

(b)

Figure 6: $(\mathrm{a}, \mathrm{b})$ The initial condition and the reference solution at $t=10^{6} h^{4}$, respectively.

TABLE 7: Errors and convergence rates for the numerical results of the LSS at the final time $T=10^{6} h^{4}$. Here, $h=1 / 128$ is used.

\begin{tabular}{lccccccc}
\hline$\Delta t$ & $10000 h^{4}$ & Rate & $5000 h^{4}$ & Rate & $2500 h^{4}$ & Rate & $1250 h^{4}$ \\
\hline$\|e\|_{2}$ & $4.287 \mathrm{e}-5$ & 0.994 & $2.153 e-5$ & 0.997 & $1.079 e-5$ & 0.998 & $5.400 e-6$ \\
$\|e\|_{\infty}$ & $5.522 e-5$ & 0.990 & $2.782 e-5$ & 0.995 & $1.397 e-5$ & 0.997 & $7.000 e-6$ \\
\hline
\end{tabular}

TABLE 8: Errors and convergence rates for the numerical results of the CN scheme at the final time $T=10^{6} h^{4}$. Here, $h=1 / 128$ is used.

\begin{tabular}{lccccccc}
\hline$\Delta t$ & $10000 h^{4}$ & Rate & $5000 h^{4}$ & Rate & $2500 h^{4}$ & Rate & $1250 h^{4}$ \\
\hline$\|e\|_{2}$ & $1.911 e-9$ & 2.000 & $4.780 e-10$ & 2.000 & $1.190 e-10$ & 1.999 & $3.000 e-11$ \\
$\|e\|_{\infty}$ & $2.860 e-9$ & 2.000 & $7.150 e-10$ & 1.998 & $1.790 e-10$ & 1.955 & $4.600 e-11$ \\
\hline
\end{tabular}

Note that the final time $T$ is set to $10^{6} h^{4}$ for ease of computation in the three-dimensional space. Figures 6(a) and 6(b) show the initial condition and the reference solution at $t=10^{6} h^{4}$, respectively.

The discrete $l_{2}$-norm and the discrete maximum norm are simply extended as follows:

$$
\begin{aligned}
\|e\|_{2} & =\sqrt{\frac{1}{N_{x} N_{y} N_{z}} \sum_{i=1}^{N_{x}} \sum_{j=1}^{N_{y}} \sum_{k=1}^{N_{z}} e_{i j k}^{2},} \\
\|e\|_{\infty} & =\max _{1 \leq i \leq N_{x} 1 \leq j \leq N_{y} 1 \leq k \leq N_{z}} e_{i j k},
\end{aligned}
$$

respectively. Tables 7 and 8 list errors and convergence rates for the numerical results at $t=10^{6} h^{4}$. Tables 7 and 8 show that equations (15) and (16) are of first-order and of secondorder in time, respectively.

\section{Conclusions}

In this work, we proposed a very simple benchmark problem, which is a cosine function as the initial condition for the numerical methods of the $\mathrm{CH}$ equations. For the numerical solution, we used RK4 for the temporal integration. Using the proposed benchmark problem solution, we performed the convergence tests for LSS and CN schemes and obtained the expected first-order and second-order convergence rates in the one-, two-, and three-dimensional $\mathrm{CH}$ equations, respectively. The proposed approach is less biased and provides more rational results compared to conventional self-convergence tests. In future work, we will 
design simple benchmark problems for other nonlinear partial differential equations such as the nonlocal $\mathrm{CH}, \mathrm{AC}$, and nonlocal AC equations.

\section{Data Availability}

No data were used to support this study.

\section{Conflicts of Interest}

The authors declare no conflicts of interest.

\section{Acknowledgments}

The first author (Y. Li) was supported by the Natural Science Basic Research Plan in Shaanxi Province of China (2016JQ1024) and by National Natural Science Foundation of China (nos. 11601416 and 11631012). This work was supported by the Brain Korea 21 FOUR Project. The corresponding author (J. Kim) was supported by the Basic Science Research Program through the National Research Foundation of Korea (NRF) funded by the Ministry of Education (NRF-2019R1A2C1003053).

\section{References}

[1] J. W. Cahn and J. E. Hilliard, "Free energy of a nonuniform system. I. Interfacial free energy," The Journal of Chemical Physics, vol. 28, no. 2, pp. 258-267, 1958.

[2] J. W. Cahn, "On spinodal decomposition," Acta Metallurgica, vol. 9, no. 9, pp. 795-801, 1961.

[3] D. Lee, J.-Y. Huh, D. Jeong, J. Shin, A. Yun, and J. Kim, "Physical, mathematical, and numerical derivations of the Cahn-Hilliard equation," Computational Materials Science, vol. 81, pp. 216-225, 2014.

[4] X. Zhang, A. Takezawa, and Z. Kang, "A phase-field based robust topology optimization method for phononic crystals design considering uncertain diffuse regions," Computational Materials Science, vol. 160, pp. 159-172, 2019.

[5] J. Shin, H. G. Lee, and J.-Y. Lee, "Unconditionally stable methods for gradient flow using Convex Splitting RungeKutta scheme," Journal of Computational Physics, vol. 347, pp. 367-381, 2017.

[6] H. Liu, A. Cheng, H. Wang, and J. Zhao, "Time-fractional Allen-Cahn and Cahn-Hilliard phase-field models and their numerical investigation," Computers \& Mathematics with Applications, vol. 76, no. 8, pp. 1876-1892, 2018.

[7] H. U. Rehman, N. Ullah, M. I. Asjad, and A. Akgül, "Exact solutions of convective-diffusive Cahn-Hilliard equation using extended direct algebraic method," Numerical Methods for Partial Differential Equations, pp. 1-16, 2020.

[8] M. Zanetti, V. Ruggiero, and M. Miranda Jr, "Numerical minimization of a second-order functional for image segmentation," Communications in Nonlinear Science and Numerical Simulation, vol. 36, pp. 528-548, 2016.

[9] P. J. Strasser, G. Tierra, B. Dünweg, and M. LukácováMedvid'ová, "Energy-stable linear schemes for polymer-solvent phase field models," Computers \& Mathematics with Applications, vol. 77, pp. 125-143, 2018.

[10] A. Shah, M. Sabir, and S. Ayub, "An adaptive time-stepping scheme for the numerical simulation of Cahn-Hilliard equation with variable mobility," Journal of Applied Mathematics and Mechanics, vol. 99, no. 7, 2019.
[11] Q. Li, L. Mei, and B. You, “A second-order, uniquely solvable, energy stable BDF numerical scheme for the phase field crystal model," Applied Numerical Mathematics, vol. 134, pp. 40-65, 2018.

[12] W. Feng, Z. Guo, J. S. Lowengrub, and S. M. Wise, "A massconservative adaptive FAS multigrid solver for cell-centered finite difference methods on block-structured, locally-cartesian grids," Journal of Computational Physics, vol. 352, pp. 463-497, 2018.

[13] P. Colli, G. Gilardi, and J. Sprekels, "A distributed control problem for a fractional tumor growth model," Mathematics, vol. 7, no. 9, p. 792, 2019.

[14] J. Kim, S. Lee, Y. Choi, S. M. Lee, and D. Jeong, "Basic principles and practical applications of the Cahn-Hilliard equation," Mathematical Problems in Engineering, vol. 2016, Article ID 9532608, 11 pages, 2016.

[15] Z. Weng, S. Zhai, and X. Feng, "Analysis of the operator splitting scheme for the Cahn-Hilliard equation with a viscosity term," Numerical Methods for Partial Differential Equations, vol. 35, no. 6, pp. 1-22, 2019.

[16] Z. Guan, J. Lowengrub, and C. Wang, "Convergence analysis for second-order accurate schemes for the periodic nonlocal Allen-Cahn and Cahn-Hilliard equations," Mathematical Methods in the Applied Sciences, vol. 40, no. 18, pp. 6836-6863, 2017.

[17] X. Zhao, "Fourier spectral approximation to global attractor for 2D convective Cahn-Hilliard equation," Malaysian Mathematical Sciences Society, vol. 41, pp. 1119-1138, 2018.

[18] X. Li, Z. Qiao, and H. Zhang, "A second-order convex splitting scheme for a Cahn-Hilliard equation with variable interfacial parameters," Journal of Computational Mathematics, vol. 35, no. 6, pp. 693-710, 2017.

[19] S. Lee, C. Lee, H. G. Lee, and J. Kim, "Comparison of different numerical schemes for the Cahn-Hilliard equation," Journal of the Korea Society for Industrial and Applied Mathematics, vol. 17, no. 3, pp. 197-207, 2013.

[20] A. M. Jokisaari, P. W. Voorhees, J. E. Guyer, J. Warren, and O. G. Heinonen, "Benchmark problems for numerical implementations of phase field models," Computational Materials Science, vol. 126, pp. 139-151, 2017.

[21] D. Jeong, Y. Choi, and J. Kim, "A benchmark problem for the two- and three-dimensional Cahn-Hilliard equations," Communications in Nonlinear Science and Numerical Simulation, vol. 61, pp. 149-159, 2018.

[22] J. M. Church, Z. Guo, P. K. Jimack et al., "High accuracy benchmark problems for allen-Cahn and Cahn-Hilliard dynamics," Communications in Computational Physics, vol. 26, no. 4, pp. 947-972, 2019.

[23] D. Jeong, Y. Li, C. Lee, J. Yang, Y. Choi, and J. Kim, "Verification of convergence rates of numerical solutions for parabolic equations," Mathematical Problems in Engineering, vol. 2019, Article ID 8152136, 10 pages, 2019.

[24] D. J. Eyre, An Unconditionally Stable One-step Scheme for Gradient Systems, Unpublished article, (1998) 1-15. Available online: http://www.math.utah.edu/\%7Eeyre/research/ methods/ch_numer.ps.

[25] J. Kim, "A numerical method for the Cahn-Hilliard equation with a variable mobility," Communications in Nonlinear Science and Numerical Simulation, vol. 12, no. 8, pp. 15601571, 2007.

[26] A. Balogh, J. Banda, and K. Yagdjian, "High-performance implementation of a Runge-Kutta finite-difference scheme for the Higgs boson equation in the de Sitter spacetime," 
Communications in Nonlinear Science and Numerical Simulation, vol. 68, pp. 15-30, 2018.

[27] J. Kim, "Phase-field models for multi-component fluid flows," Communications in Computational Physics, vol. 12, no. 3, pp. 613-661, 2012.

[28] U. Trottenberg, C. Oosterlee, and A. Schüller, Multigrid, Academic Press, New York, NY, USA, 2001. 